\title{
Is the Renal Resistive Index a Marker for Revascularization in Atherosclerotic Renal Artery Stenosis?
}

\author{
Lalitha Padmanabha Vemireddy ${ }^{1}$, Grace W. Ying ${ }^{1}$, Ammar Aqeel ${ }^{1}$, Shaji Baig ${ }^{2}$, Venkata Buddharaju ${ }^{3}$ \\ 1. Internal Medicine, Chicago Medical School Internal Medicine Residency Program, Northwestern McHenry Hospital, \\ Chicago, USA 2. Internal Medicine, Northwestern Medicine McHenry Hospital, Rosalind Franklin University of \\ Medicine and Science, McHenry, USA 3. Nephrology, Northwestern Medicine McHenry Hospital, Rosalind Franklin \\ University of Medicine and Science, McHenry, USA
}

Corresponding author: Lalitha Padmanabha Vemireddy, lalitha.vemireddy@rosalindfranklin.edu

\begin{abstract}
Renal artery stenosis (RAS) is one of the major causes of resistant/malignant hypertension. It can be described as atherosclerotic or non-atherosclerotic. Atherosclerotic RAS comprises almost $90 \%$ of all RAS cases and is a prevalent disease of the elderly. Multiple risk factors contribute to atherosclerosis development, which leads to the release of renin and aldosterone, causing resistant/malignant hypertension. Early recognition is prudent but challenging as there are no early clinical signs. We believe that renal resistive index with supportive clinical, laboratory, and imaging modalities can help select revascularization patients.
\end{abstract}

Categories: Cardiology, Internal Medicine, Nephrology

Keywords: atherosclerotic renal artery, renal artery stenting, renal artery stenosis, renal resistive index

\section{Introduction}

Renal artery stenosis (RAS) is an important cause of malignant hypertension. Among the 50 million people in the United States diagnosed with hypertension, the incidence of malignant hypertension can be as high as $10 \%$ [1]. RAS is further categorized into atherosclerotic RAS (ARAS) and non-atherosclerotic RAS (N-ARAS) [2]. Atherosclerosis constitutes almost $90 \%$ of all RAS cases, with non-atherosclerotic causes making up the remaining portion [2]. With fibromuscular dysplasia being the most common cause of N-ARAS, some other contributing etiologies may include Takayasu's arteritis, Buerger's disease, thromboembolic disease, and infra-renal aneurysm [1,2]. Unlike N-ARAS, ARAS commonly occurs in the elderly and does have multiple risk factors such as hyperlipidemia, smoking, increased homocysteine levels, diabetes mellitus, family history, and old age $[1,3,4]$. Even though ARAS is only seen in $1 \%-6 \%$ of hypertension cases, it can present in as high as $50 \%$ of the elderly population with a known history of atherosclerotic disease [5]. ARAS treatment options usually consist of medical therapy, percutaneous angioplasty, or surgical intervention $[2,4,5]$. Management of RAS has been controversial as multiple studies have failed to establish one treatment therapy's superiority over another. We are presenting two cases of ARAS in patients with existing congestive

Review began 04/12/2021 Review ended 04/22/2021 Published 04/29/2021

\section{๑) Copyright 2021}

Vemireddy et al. This is an open access article distributed under the terms of the Creative Commons Attribution License CC-BY 4.0., which permits unrestricted use, distribution, and reproduction in any medium, provided the original author and source are credited. heart failure $(\mathrm{CHF})$ and discuss potential treatment challenges working with current management guidelines.

\section{Case Presentation}

\section{Case 1}

An 88-year-old female with a past medical history of hypertension, coronary artery disease, combined systolic and diastolic CHF with a left ventricular ejection fraction of $40 \%$, peripheral artery disease status post femoral bypass grafting, and diabetes mellitus type II, chronic kidney disease stage IIIB (calculated creatinine clearance of 31) presented to our hospital with complaints of dyspnea for 2-3-day duration. On presentation, the patient was found to be in a hypertensive emergency with blood pressure (BP) of 220/91 $\mathrm{mmHg}$ accompanied by signs of fluid overload. Lab studies were within normal limits. She was discharged after being treated with diuretics and anti-hypertensives. She presented again within a month with similar symptoms and BP of 188/79 mmHg. Due to recurrent flash pulmonary edema and resistant hypertension, a renal artery ultrasound was performed, which demonstrated atrophic right kidney and poorly visualized renal arteries. A subsequent renal angiogram showed $70 \%$ stenosis of the right renal artery, which was later intervened with a bare-metal stent (Figures 1,2). 


\section{Cureus}

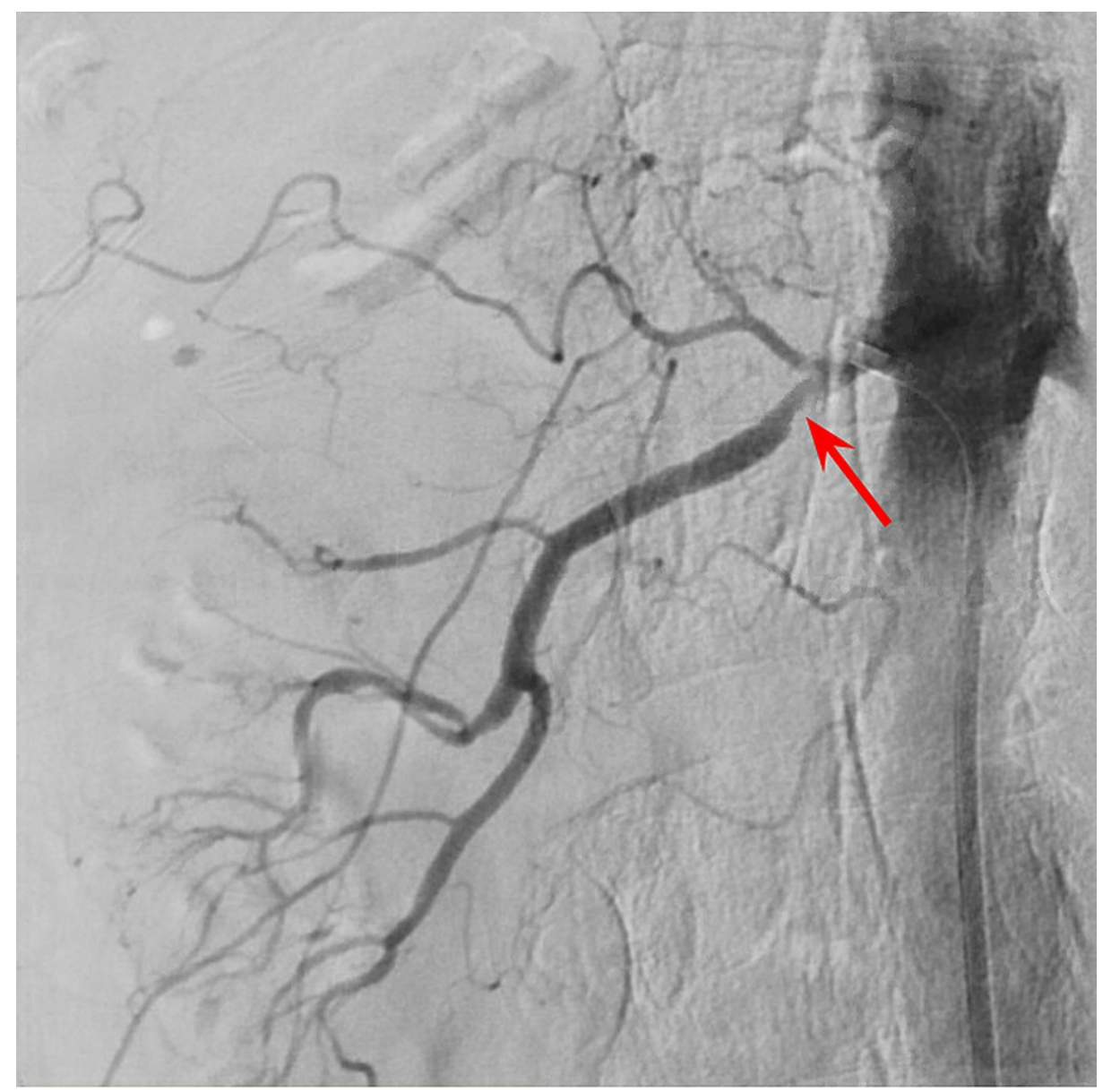

FIGURE 1: Right renal artery angiogram showing stenosis of the right renal artery (red arrow) close to the origin from descending aorta 


\section{Cureus}

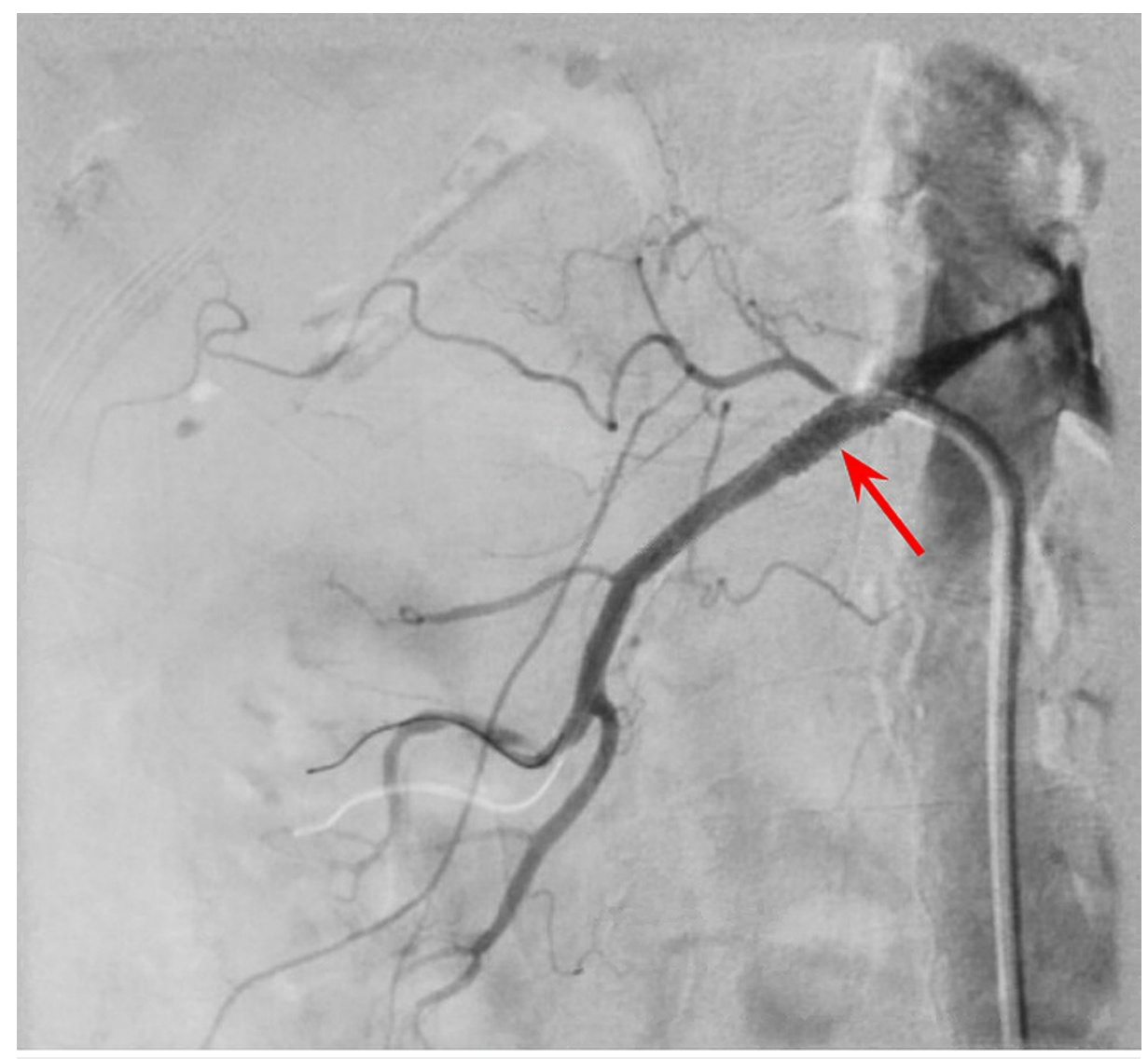

FIGURE 2: Right renal angiogram showing renal artery stent (red arrow) with no residual stenosis

Nicardipine drip was initiated for worsening BP post-operatively in addition to the continuation of diuretics. As the patient's respiratory status worsened despite being on aggressive management, her family ultimately decided and opted for hospice. Unfortunately, our patient passed away a few hours later.

\section{Case 2}

An 86-year-old female with a past medical history of hypertension, coronary artery disease status post percutaneous coronary intervention, diastolic CHF with a left ventricular ejection fraction of $69 \%$, insulindependent type II diabetes mellitus, dyslipidemia, cerebrovascular disease, chronic kidney disease stage IIIB (calculated creatinine clearance of 39) and anxiety presented to the hospital with complaints of sub-sternal chest discomfort and frontal headache for several days. She had numerous admissions in the past for refractory hypertension. Upon being on multiple antihypertensive regimens, initial vital signs on this admission were stable except for a BP of 221/88 mmHg. Computed tomographic angiography of chest/abdomen/pelvis found high-grade stenosis at the right renal artery origin with at least $50 \%$ stenosis of the left renal artery origin (Figure 3). 


\section{Cureus}

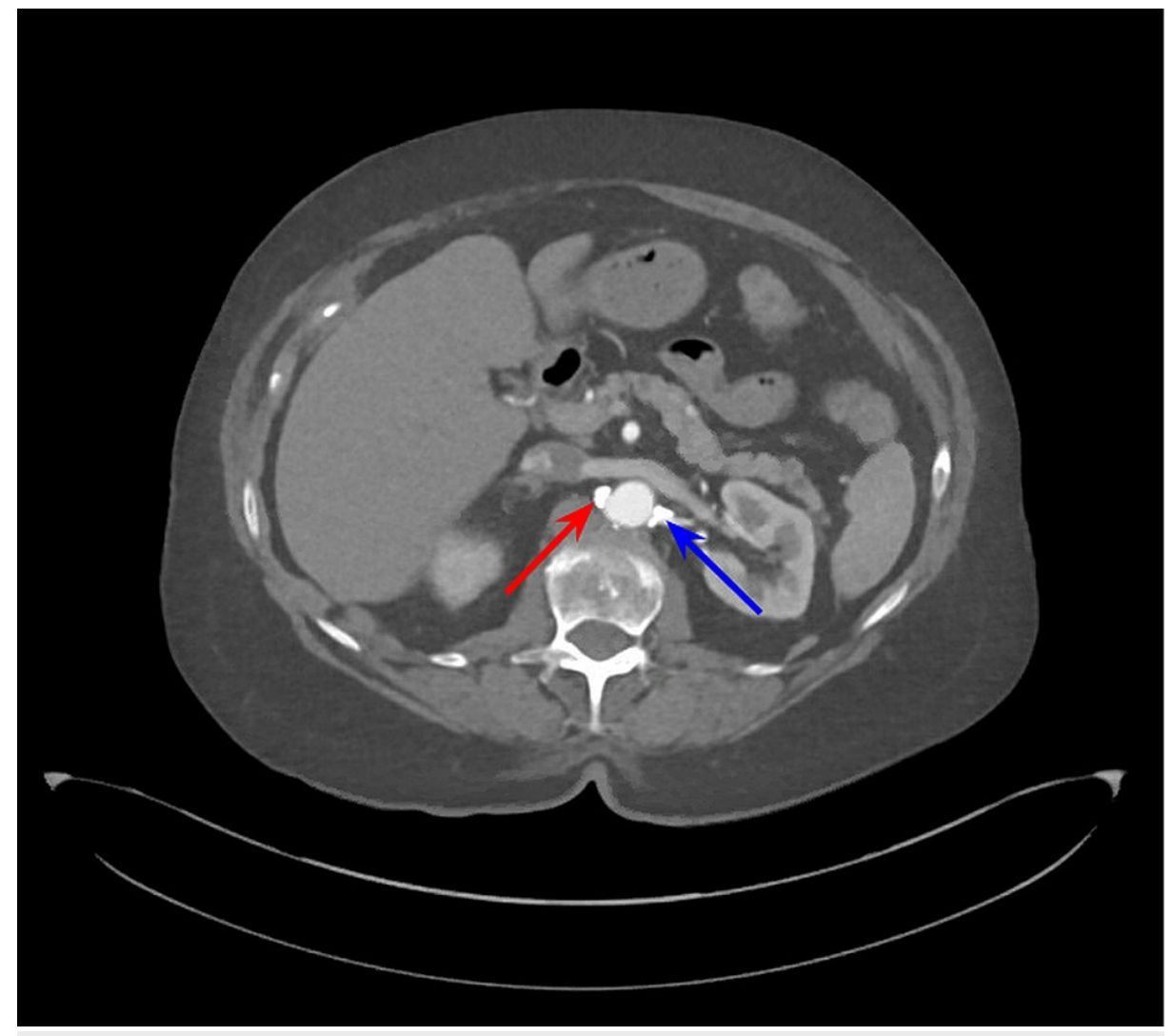

FIGURE 3: CTA abdomen demonstrating a high-grade stenosis of the right renal artery origin (red arrow) from aorta and $>50 \%$ stenosis of the left renal artery (blue arrow)

CTA - Computed tomographic angiography.

She subsequently underwent renal angiography with a right renal artery stent placement and was discharged home on several antihypertensive medications (Figures 4, 5). 


\section{Cureus}

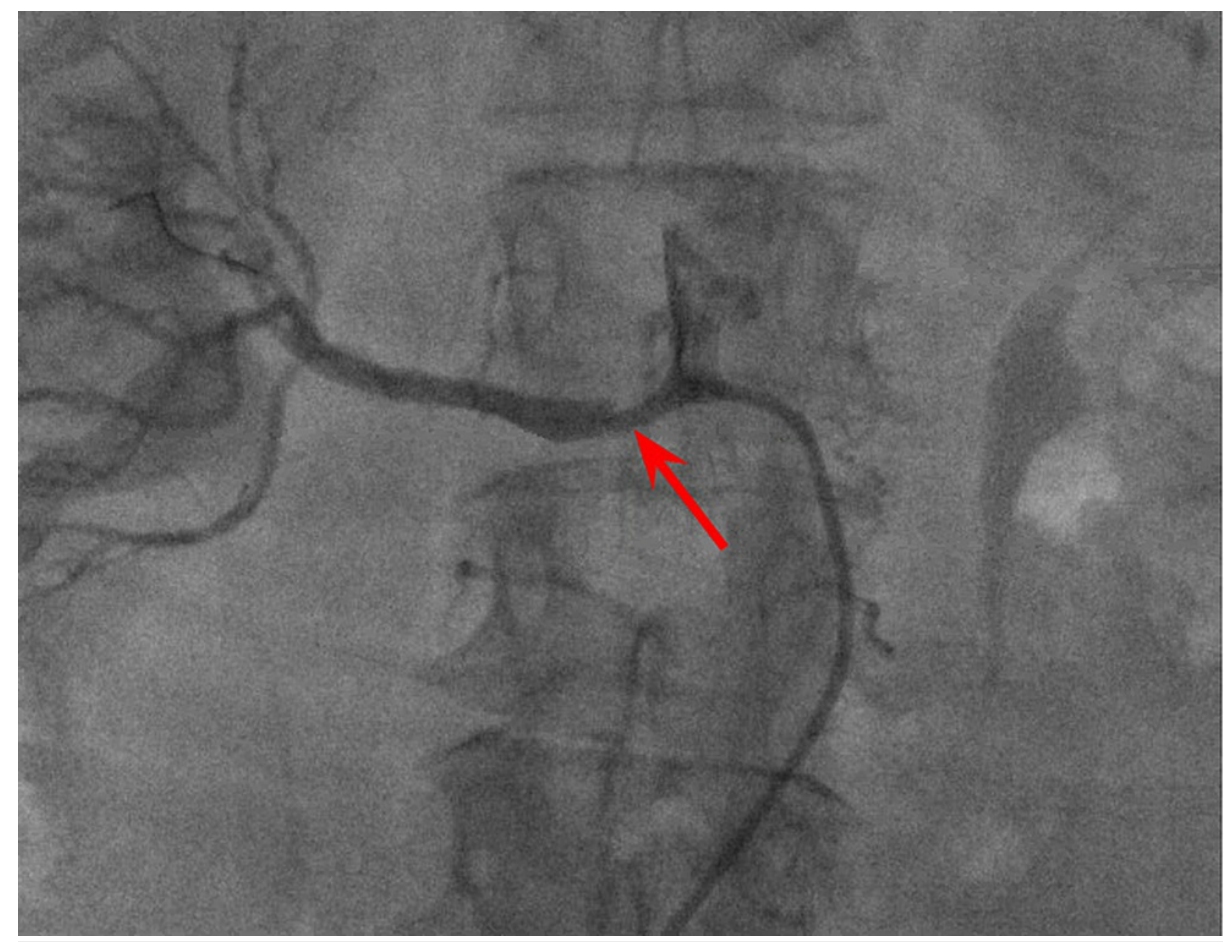

FIGURE 4: Right renal angiogram showing stenosis of the right renal artery (red arrow)

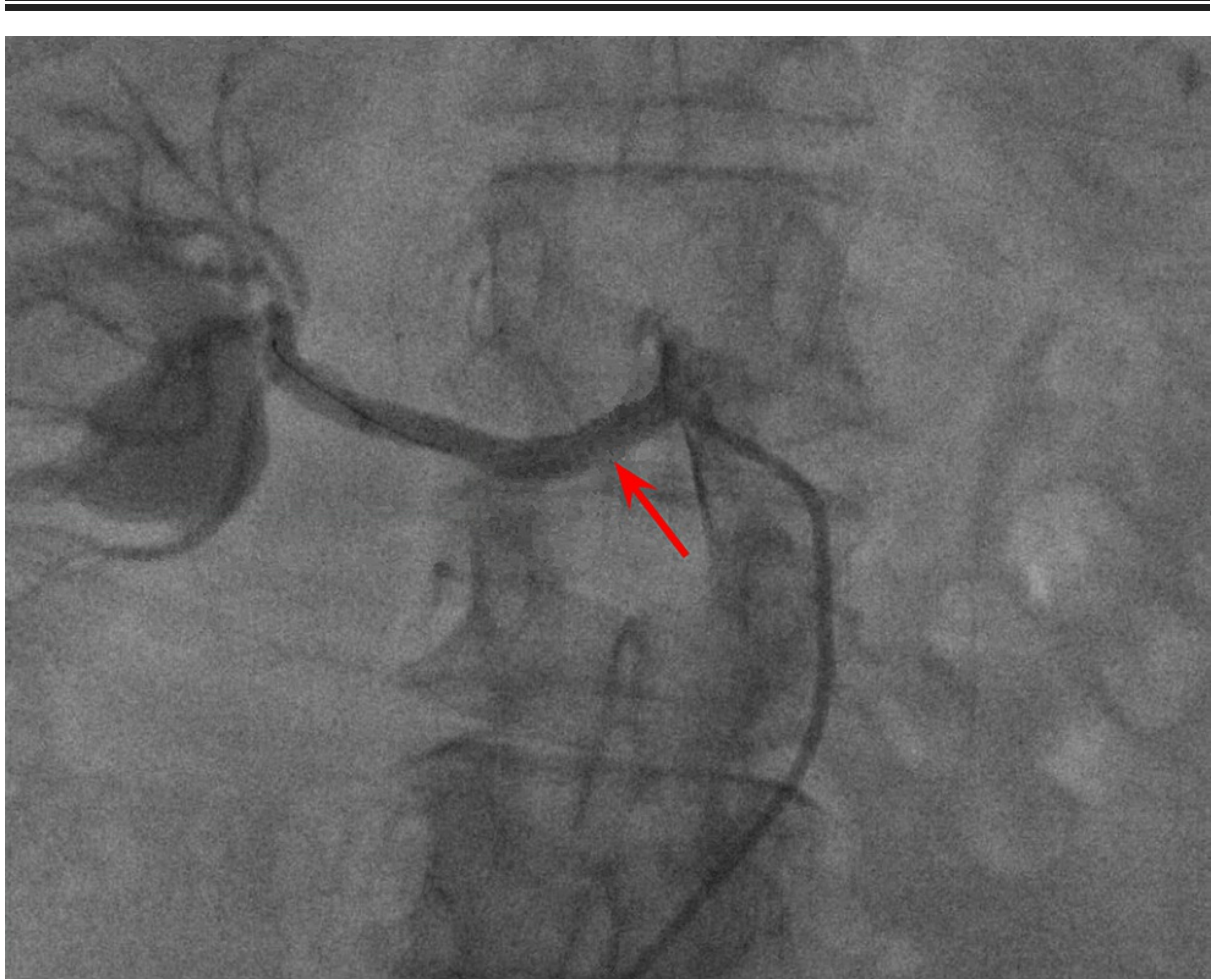

FIGURE 5: Right renal angiogram showing right renal artery with stent (red arrow) and no residual stenosis

Her BP was not effectively lowered as expected following the procedure with the BP measured 198/80 $\mathrm{mmHg}$ during the outpatient cardiology office visit despite good medication adherence. The patient was readmitted to the hospital a week later for a hypertensive emergency with a BP of $180 / 70 \mathrm{mmHg}$ and was discharged home again with numerous BP medications, including losartan, clonidine, and spironolactone. 


\section{Discussion}

As previously mentioned, ARAS constitutes almost $90 \%$ of RAS cases [2]. The primary mechanism of ARASinduced hypertension is due to activation of the renin-angiotensin-aldosterone system leading to subsequent fluid overload. Any risk factor capable of causing endovascular injury can lead to accumulation of low-density lipoprotein, macrophages, and proliferation of endothelial and smooth muscle cells forming atherosclerotic plaques. Progressive plaque build-up results in a decrease in blood flow to the kidneys, which activates the juxtaglomerular apparatus (JGA). JGA subsequently releases renin, which produces angiotensin I. Angiotensin I is further converted into angiotensin II causing vasoconstriction and aldosterone production from the adrenal glands. Aldosterone contributes to salt and water retention leading to fluid overload and hypertension [1,2].

ARAS is often suspected with the presence of one or more clinical signs. These may include resistant hypertension (failure to achieve BP control with $\geqslant 3$ antihypertensive agents of different classes), uncontrolled hypertension in patients $>50$ years of age, often with a history of carotid artery stenosis, coronary artery stenosis or peripheral arterial disease, azotemia, or worsening renal function especially after administration of angiotensin-converting enzyme inhibitors (ACEIs) or angiotensin receptor blockers (ARBs), unexplained hypokalemia, new-onset abdominal bruits, unexplained CHF, or recurrent flash pulmonary edema $[2,5]$.

ARAS diagnosis can be made using Doppler ultrasonography, computed tomographic angiography, magnetic resonance angiography, invasive angiography, or renal scintigraphy scan (Captopril Renal Scan). Each of these imaging techniques comes with its own set of advantages and disadvantages. Ultimately the imaging of choice should be determined on a case-by-case basis considering varied comorbidities among patients $[2,5,6]$.

When it comes to ARAS treatment, there are two different approaches: medical therapy and revascularization, including renal artery stenting and surgical revascularization. Medical therapy usually involves the initiation of antihypertensive agents, statins, risk factor modification like glycemic control and smoking cessation, and primary prevention with aspirin. The antihypertensive agents of choice are ACEI, ARBs, beta-blockers, and calcium channel blockers. While patients are on ACEI or ARBs, renal function should be monitored closely. On the other hand, renal artery revascularization can be achieved through balloon angioplasty with or without stenting or surgical revascularization. Several studies have shown that surgical revascularization is associated with higher rates of complication. Thus, percutaneous intervention is often preferred $[2,5,7]$.

There are three major randomized clinical trials (STAR, ASTRAL, CORAL) comparing medical therapy with stent placement in patients diagnosed with ARAS. However, all of them have failed to show a statistically significant difference regarding mortality benefit, renal function, or BP control [8-10]. A retrospective study done by Kane et al. showed no statistical difference between the medical therapy group and the angioplasty group, with the long-term need for dialysis being equally high. However, there seemed to be some shortterm improvement in renal function with angioplasty [11]. Radermacher et al. have been pivotal in measuring the renal resistive index (RRI) with Doppler ultrasound as a non-invasive measurement of renal hemodynamics. RRI, calculated as (peak systolic velocity - end-diastolic velocity)/peak systolic velocity, is associated with various cardiovascular events and mortality in elderly, hypertensive, and diabetic patients. As part of the screening process to determine the likelihood to benefit from intervention, Radermacher et al. came up with a list of criteria including age $>65$, pulse pressure of at least $70 \mathrm{mmHg}, \mathrm{CrCl}<40 \mathrm{~mL} / \mathrm{min}$, urine protein excretion of at least 1 gram per day, hyperuricemia, coronary artery disease, peripheral artery disease, and cerebrovascular disease [12].

Furthermore, Soulez et al. also proposed some valuable predictors of outcome after renal angioplasty. These include measurements of kidney length and unilateral and bilateral RRI before and after the administration of captopril. For example, bilateral renal artery RRI $<0.75$ before captopril administration and unilateral RRI $<0.70$ after captopril administration; or bilateral renal artery RRI $<0.75$ before captopril administration and kidney length measuring longer than $90 \mathrm{~mm}$ have been found to have favorable outcome [13]. A study done by Davies et al. analyzed renal parenchymal preservation and that patients who had parenchymal losses were found to have higher RRI. This was correlated with worse clinical outcomes and faster progression to dialysis [14]. Zeller et al. divided 176 patients into three groups based on RRI (RI $<0.7$, RI 0.7-0.8, RI >0.8), and it was found that the improvement in renal function and the reduction in BP were the same across all study groups [15].

Considering the factors Radermacher et al. proposed, our two patients met at least half of the criteria, so we believe that the intervention would not have changed the outcome in terms of BP control, renal function, or overall mortality. The current guidelines recommend an invasive strategy for those not responding well to optimal medical therapy or are subject to recurrent CHF exacerbations or flash pulmonary edema. Stricter criteria based on further clinical trials involving age, kidney function, diabetes, other comorbid conditions, laboratory data, RRI, etc. need to be developed. Based on these factors, suitable candidates should be selected who would benefit from revascularization. 


\section{Conclusions}

ARAS is a common cause of resistant/malignant hypertension especially in the elderly. Medical therapy still remains the mainstay of treatment while some patients require revascularization. As multiple randomized clinical trials have proven that revascularization does not improve mortality, renal function, or blood pressure, it is of utmost importance to develop a specific set of criteria including RRI and various clinical, laboratory, and imaging modalities to select patients who would benefit from revascularization.

\section{Additional Information \\ Disclosures}

Human subjects: Consent was obtained or waived by all participants in this study. Conflicts of interest: In compliance with the ICMJE uniform disclosure form, all authors declare the following: Payment/services info: All authors have declared that no financial support was received from any organization for the submitted work. Financial relationships: All authors have declared that they have no financial relationships at present or within the previous three years with any organizations that might have an interest in the submitted work. Other relationships: All authors have declared that there are no other relationships or activities that could appear to have influenced the submitted work.

\section{References}

1. Bokhari MR, Bokhari SRA: Renal Artery Stenosis. StatPearls [Internet]. StatPearls Publishing, Treasure Island, FL; 2020.

2. Weber BR, Dieter RS: Renal artery stenosis: epidemiology and treatment. Int J Nephrol Renovasc Dis. 2014, 7:169-81. 10.2147/IJNRD.S40175

3. Kalra PA, Guo H, Kausz AT, et al.: Atherosclerotic renovascular disease in United States patients aged 67 years or older: risk factors, revascularization, and prognosis. Kidney Int. 2005, 68:293-301. 10.1111/j.15231755.2005.00406.x

4. Paraskevas KI, Hamilton G, Cross JM, Mikhailidis DP: Atherosclerotic renal artery stenosis: association with emerging vascular risk factors. Nephron Clin Pract. 2008, 108:c56-66. 10.1159/000112556

5. Lao D, Parasher PS, Cho KC, Yeghiazarians Y: Atherosclerotic renal artery stenosis--diagnosis and treatment. Mayo Clin Proc. 2011, 86:649-57. 10.4065/mcp.2011.0181

6. Hirsch AT, Haskal ZJ, Hertzer NR, et al.: ACC/AHA 2005 Practice Guidelines for the management of patients with peripheral arterial disease (lower extremity, renal, mesenteric, and abdominal aortic): a collaborative report from the American Association for Vascular Surgery/Society for Vascular Surgery, Society for Cardiovascular Angiography and Interventions, Society for Vascular Medicine and Biology, Society of Interventional Radiology, and the ACC/AHA Task Force on Practice Guidelines (Writing Committee to Develop Guidelines for the Management of Patients With Peripheral Arterial Disease): endorsed by the American Association of Cardiovascular and Pulmonary Rehabilitation; National Heart, Lung, and Blood Institute; Society for Vascular Nursing; TransAtlantic Inter-Society Consensus; and Vascular Disease Foundation. Circulation. 2006, 113:e463-654. 10.1161/CIRCULATIONAHA.106.174526

7. Weibull H, Bergqvist D, Bergentz SE, Jonsson K, Hulthén L, Manhem P: Percutaneous transluminal renal angioplasty versus surgical reconstruction of atherosclerotic renal artery stenosis: a prospective randomized study. J Vasc Surg. 1993, 18:841-52. 10.1067/mva.1993.45062

8. Cooper CJ, Murphy TP, Cutlip DE, et al.: Stenting and medical therapy for atherosclerotic renal-artery stenosis. N Engl J Med. 2014, 370:13-22. 10.1056/NEJMoa1310753

9. Bax L, Woittiez AJ, Kouwenberg HJ, et al.: Stent placement in patients with atherosclerotic renal artery stenosis and impaired renal function: a randomized trial. Ann Intern Med. 2009, 150:840-8, W150-1. 10.7326/0003-4819-150-12-200906160-00119

10. Wheatley K, Ives N, Gray R, et al.: Revascularization versus medical therapy for renal-artery stenosis . N Engl J Med. 2009, 361:1953-62. 10.1056/NEJMoa0905368

11. Kane GC, Xu N, Mistrik E, Roubicek T, Stanson AW, Garovic VD: Renal artery revascularization improves heart failure control in patients with atherosclerotic renal artery stenosis. Nephrol Dial Transplant. 2010, 25:813-20. 10.1093/ndt/gfp393

12. Radermacher J, Chavan A, Bleck J, et al.: Use of Doppler ultrasonography to predict the outcome of therapy for renal-artery stenosis. N Engl J Med. 2001, 344:410-7. 10.1056/NEJM200102083440603

13. Soulez G, Therasse E, Qanadli SD, et al.: Prediction of clinical response after renal angioplasty: respective value of renal Doppler sonography and scintigraphy. AJR Am J Roentgenol. 2003, 181:1029-35. 10.2214/ajr.181.4.1811029

14. Davies MG, Saad WE, Bismuth J, Naoum JJ, Peden EK, Lumsden AB: Renal parenchymal preservation after percutaneous renal angioplasty and stenting. J Vasc Surg. 2010, 51:1222-9; discussion 1229. 10.1016/j.jvs.2009.09.050

15. Zeller T, Müller C, Frank U, et al.: Stent angioplasty of severe atherosclerotic ostial renal artery stenosis in patients with diabetes mellitus and nephrosclerosis. Catheter Cardiovasc Interv. 2003, 58:510-5. 10.1002/ccd.10435 